\title{
SDE Simulation in One Click: Fiction or Reality?
}

\author{
Alexandra Barysheva, Alexander Markov \\ School of Nuclear Science \& Engineering \\ National Research Tomsk Polytechnic university \\ Tomsk, Russia \\ melnikae@tpu.ru, markovas@tpu.ru
}

\begin{abstract}
Stochastic differential equations (further referred to as SDEs) and the models based on SDE are widely used to describe stochastic processes in virtually any area of human activity, such as biology or finance. Unlike an analytical approach to solving SDE, the simulation methods allow to significantly increase the range of practical problems, which examples are given in the paper. Capture III describes the result of the comparative analysis of existing programming tools for SDE simulation, their advantages and shortcomings. It was shown that none of the existing tools completely meet the requirements formulated in Chapter III for the tool's functionality used for building a simulation model.
\end{abstract}

Key words-stochastic differential equations, discretization scheme, Monte-Carlo simulation

\section{INTRODUCTION}

Advances in mathematical theory gave impetus to the development of specific lines of research that study natural phenomena and processes whose dynamics are impossible to predict. Practical application was found for two fundamentally different theories: chaos theory and probability theory. Regardless of its name, chaos theory implies that unpredictable processes are deterministic, and that the path of such process, including its future path, can be absolutely determined by a known law (function). Only the initial data (starting point of the process) is a source of uncertainty [1].

A more widespread approach to analyzing stochastic processes is using methods of probability theory, which refers to these processes as stochastic ${ }^{1}$, and the occurrence of this or that event is considered in terms of probability. Examples of stochastic processes can be seen in virtually any area of human activity, such as biology, chemistry, epidemiology, mechanics, economics, and finance. "How will a financial asset's price change?", "How many spots will the Sun have?" or "How will the population of two competing animal species change?" these are examples of the broad range of practical problems that can be resolved by simulating stochastic processes.

\section{USING STOCHASTIC DIFFERENTIAL EQUATIONS TO DESCRIBE STOCHASTIC PROCESSES}

Among the variety of models used to describe stochastic processes, particular attention shall be given to the models based on stochastic differential equations. As shown by applied research, [2], [3], [4], [5], such models are capable of replicating the properties of many observed processes in sufficiently high detail. Let us introduce several examples of SDE-based models used to deal with real-life problems in different areas of research.

\footnotetext{
${ }^{1}$ a family of random variables indexed by a certain parameter, most frequently time or a coordinate
}

\section{A. Mathematical finance}

The Heston model, intended to describe the joint dynamics of an underlying asset and its volatility, is efficiently used in mathematical finance to deal with the issues of hedging and derivative pricing. Aside from the randomness present in the change of the underlying asset, volatility is also described by a stochastic process. The model is described as follows:

$$
\begin{aligned}
& d S_{t}=\mu S_{t} d t+\sqrt{v_{t}} S_{t} d W_{t}^{S} \\
& d v_{t}=k\left(\theta-v_{t}\right) d t+\xi \sqrt{v_{t}} d W_{t}^{v}
\end{aligned}
$$

where $d W_{t}^{S}, d W_{t}^{v}$ are correlated Wiener processes, are the price and volatility of the underlying, and $\theta, \xi^{-}$are the model parameters [6].

\section{B. Physics}

The process of solar activity, or that of the change of the number of sunspots, is also considered stochastic because to this moment no theoretical explanation has been found for the periodicity of its dynamics. In 1982, Matyas Arato suggested the following model to describe this process [7]:

$$
d\left[\begin{array}{l}
x_{t} \\
y_{t}
\end{array}\right]=\left[\begin{array}{cc}
0 & 1 \\
-a_{2} & -a_{1}
\end{array}\right]\left[\begin{array}{l}
x_{t} \\
y_{t}
\end{array}\right] d t+\left[\begin{array}{l}
0 \\
c
\end{array}\right] d W_{t}
$$

where $x_{t}$ is the number of sunspots, $W_{t}$ is a standard Wiener process, and $a_{1}, a_{2}, c$ are constants.

\section{Biology/chemistry}

The model of calculating the populations of competing species by Vito Volterra [8] and the model of chemical reactions suggested by Alfred J. Lotka are similar in form and meaning. Suggesting that in particular conditions, the processes of chemical reactions (or coexistence of two competing species) $\xi^{\mathbf{1}}, \xi^{\mathbf{z}}$ can be considered independent white noises, the model equation can be expressed as follows:

$$
\begin{aligned}
& \frac{d x_{t}}{d t}=\left(k_{1}^{\prime}+a_{1} y_{t}\right) x_{t}+\sigma_{1} x_{t} \xi_{t}^{1} \\
& \frac{d y_{t}}{d t}=\left(k_{2}^{\prime}+a_{2} x_{t}\right) y_{t}+\sigma_{2} y_{t} \xi_{t}^{2}
\end{aligned}
$$

where $a_{1}, \sigma_{1}, a_{2}, \sigma_{2}$ are constants, and $x_{t}, y_{t}$ is the concentration of reagents (population of the competing species) [7].

All of the models described above are a subclass of mathematical diffusion models, whose general form is expressed as follows:

$$
\begin{aligned}
& \frac{d x_{t}}{d t}=a\left(x_{t}, u(t), t\right)+\sum\left(x_{t}, t\right) r_{t}, \\
& x_{0}=x(0, w)
\end{aligned}
$$


where $x_{t}$ is the system state vector, $u(t)$ is the control action, $r(t)$ is the stochastic random excitation representing a stochastic vector process, $a$ are matrix functions of eigen arguments, and $x_{0}$ is the random vector of the initial conditions [7].

Covering a broad range of practical problems, diffusion models are the most popular in mathematical finance, electrodynamics, and control theory. However, it should be noted that processes with jump-process structure are often described using diffusion-jump process models:

$$
d x_{t}=a_{t} d t+\sum_{i=1}^{m} b_{t}^{i} d f_{t}^{i}+\int q(t, x) \mu(d t, d x),
$$

where $\mu(d t, d x)$ is an integer random number, $f_{t}^{i}$ are independent Wiener processes, independent from the random number's values. An example of such model is the generalized Black-Scholes model, where jump-process behavior is described with a Poisson process [9].

\section{Issues in studying stochastic process properties}

From the practical point of view, one of the main ideas of building a system's model is studying the unobservable properties of a process. There are two common approaches for studying the properties of stochastic processes:

1. Analytical approach;

2. Simulation.

The analytical method is preferred because it allows to obtain an accurate solution for both the SDE and/or its properties. Use of the analytical method does not require the use of discrete time, since the mathematical apparatus allows to operate continuous processes, which ensures the accuracy of all conclusions. A significant drawback of this method is its limited use, as it is only applicable to relatively simple models. In reality, researchers tend to use simulation modeling methods, whose obvious advantage lies in their broadness and versatility of use, which, in its turn, allows to significantly increase the range of practical problems that can be solved using SDEs. In many cases, simulation modeling is the only possible way of studying the properties of a system or a process. Along with a significant advantage in applicability, the technique of simulation modeling has a number of shortcomings:

1. Simulation modeling requires a process to be sampled (both in time and values), since it is carried out using a computer, and the existing computers are unable to operate continuous processes;

2. Simulation modeling is resource-consuming because it does not imply generating a large number of process paths;

3. It is hard to guarantee the specified accuracy of solution because the range of error depends on a number of factors (for example the choice of discretization scheme and the number of paths).

Regardless of the existing drawbacks, the versatility of this method has led to the fact that today simulation approach is the most widespread approach to studying properties of stochastic processes.
However, researchers often face a number of difficulties when attempting to implement a simulation model in practice. First of all comes the choice of a suitable model. Even though at the stage of studying the properties of a process it is assumed that the model's form is somehow determined by the researcher, the existence of the qualitative and quantitative criteria for comparing alternatives can help to perform comparative analysis relatively fast and make a justified choice of the model's type.

Second comes the choice of a technique to discretize the process. As was mentioned above, in order to simulate continuous processes using a computer, it is necessary to switch from continuous time to discrete time, which entails two very important questions:

1. How to choose the interval of discretization;

2. Which scheme of discretization is the most suitable for the SDE type in question.

In addition, while choosing the model type, discretization method, and discretization interval, one should not forget that any stochastic process has its apriori properties that must also be observed in the paths built using the model. For example, applying Euler discretization scheme directly to the BlackScholes model leads to nonfulfillment of the apriori property of outlier non-negativity [10]. However, if the same sheme is applied to logarithmic prices of the outlier, it allows to avoid negative price values. Another example of change of a simulation model's quality depending on the choice of the discretization scheme is shown in the table below. Here, the cost estimation error obtained using different discretization schemes is calculated for an option, whose underlying asset price changes according to the Heston model.

\section{TABLE 1. ABSOLUTE OPTION COST ESTIMATION ERROR FOR THE HESTON MODEL OBTAINED USING DIFFERENT} DISCRETIZATION SCHEMES [11], [12]

\begin{tabular}{|l|c|c|c|c|c|}
\hline $\begin{array}{c}\text { Period } \\
\text { (year) }\end{array}$ & $\begin{array}{c}\text { Scheme } \\
\mathbf{1}\end{array}$ & $\begin{array}{c}\text { Scheme } \\
\mathbf{2}\end{array}$ & $\begin{array}{c}\text { Scheme } \\
\mathbf{3}\end{array}$ & $\begin{array}{c}\text { Scheme } \\
\mathbf{4}\end{array}$ & $\begin{array}{c}\text { Scheme } \\
\mathbf{5}\end{array}$ \\
\hline 1 & $-6,39$ & $-57,65$ & $-1,29$ & $-1,02$ & $-0,3$ \\
\hline $1 / 2$ & $-3,68$ & $-32,98$ & $-0,61$ & $-0,31$ & $-0,26$ \\
\hline $1 / 4$ & $-2,04$ & $-18,43$ & $-0,32$ & $-0,04$ & $-0,16$ \\
\hline $1 / 8$ & $-1,05$ & $-10,12$ & $-0,23$ & $-0,002$ & $-0,14$ \\
\hline $1 / 16$ & $-0,51$ & $-5,33$ & $-0,16$ & 0,004 & $-0,09$ \\
\hline
\end{tabular}

As can be seen, the choice of the model, scheme and interval of discretization directly affects the quality and accuracy of the simulation model. Therefore, in reality, on the stage of planning the construction of a simulation model, a researcher has to carry out a number of operations to select the optimal algorithm that will allow to obtain high-quality paths of a stochastic process while preserving all of its apriori properties.

\section{STOCHASTIC PROCESS MODELING TOOLS}

From a researcher's perspective, it would be convenient to have at one's disposal a tool that would allow to analyze a system, compare existing models, implement various discretization schemes, as well as estimate different properties of a stochastic process. What functionality should such a tool 
possess? Let us consider the optimal functionality of a hypothetical software tool that would meet all the requirements.

1. User-friendly interface. Since application of SDE-based models is not limited to engineering and natural sciences, the user interface must be intuitive for any user, be it a physics engineer or a sociology student. In-depth knowledge of programming should not be necessary to use such hypothetical tool;

2. The option of configuring the type of the model (type of SDE) aside from just choosing proposed models from a list. This would substantially broaden the area of this tool's application and make it universal;

3. The possibility to select and configure the interval of a continuous stochastic process (uniform and non-uniform grid), since with some processes the use of a non-uniform grid does not only increase the quality of the obtained paths, but also reduces the time consumed.

4. A broad functionality to select and configure discretization schemes and the criteria for their comparison (for example rate of convergence, resource consumption, etc.)

5. Because building a model is not an end in itself and a researcher is ultimately interested in the properties of the stochastic process under study, the tool must possess a sufficiently broad and flexible functionality that would allow to estimate not only the probable paths of the process, but, according to the user's desire, its characteristics and properties as well.

6. The option to simulate several stochastic processes with a set correlation structure. For example, two stochastic processes described by the Heston model.

Availability of a tool that possessed the functionality described above would significantly decrease the time of the initial analysis and selecting an optimal algorithm (including the scheme of discretization) to build a simulation model, and would allow the use of simulation approach by people who do not have substantial programming skills, thus allowing them to independently analyze the properties of stochastic processes without resorting to help from specialists. The relevance of developing such tool is corroborated not only by the broad range of the practical problems that can be solved with its help, but also by the current competition on the market of various software packages for stochastic process modeling. Most of the existing packages represent implementations of some particular cases of specific models, for example, Black-Scholes model simulation can be easily implemented in R (jrvFinance [13] and NMOF [14]) or Matlab environments (Finance Tool). However, the author believes the following software products to be the most interesting implementations of simulation approach techniques:

1. R/ Yuima [15]

2. Matlab/ Econometric/Finance Tool [16]

3. Python/ sdeint [17]

The "Yuima" packages developed in $\mathrm{R}$ encapsulates the "sde" package by Stefano Maria Iacus, which allows to simulate one-dimensional stochastic processes using diffusion models. "Yuima" is one of the most versatile of the existing software products, encompassing a broad range of SDE-based models. It allows its user to set the desired type of SDE for analysis and modeling. This package is freely available, which is another substantial advantage. The software solutions for SDE model simulation designed by Matlab team are assembled in "Econometric" and "Finance Tools". With these programs, the user can select a model from a limited available list (for example Black-Scholes, Heston, CIR models [18]) and also use a standard Euler discretization scheme [14], as well as using the Milstein method, though this is the end of discretization scheme options. The possibilities of the "sdeint" Python library are more limited in comparison with the software already described. Let us analyze the selected software products according to the criteria defined above.

\section{TABLE 2. COMPARISON OF THE EXISTING SDE SIMULATION} MODELING PACKAGES

\begin{tabular}{|l|c|c|c|}
\hline \multicolumn{1}{|c|}{ Functionality } & $\begin{array}{r}\text { R/ } \\
\text { Yuima }\end{array}$ & $\begin{array}{c}\text { Matlab/ } \\
\text { Finance } \\
\text { Toolbox }\end{array}$ & $\begin{array}{c}\text { Python/ } \\
\text { sdeint }\end{array}$ \\
\hline Simple interface & - & - & - \\
\hline $\begin{array}{l}\text { Possible to configure/select model } \\
\text { type }\end{array}$ & + & $+*$ & - \\
\hline $\begin{array}{l}\text { Possible to configure discretization } \\
\text { interval }\end{array}$ & - & - & - \\
\hline $\begin{array}{l}\text { Possible to select/configure } \\
\text { discretization scheme }\end{array}$ & - & $+*$ & - \\
\hline $\begin{array}{l}\text { Possible to estimate user-selected } \\
\text { process properties }\end{array}$ & - & - & - \\
\hline $\begin{array}{l}\text { Simulation of several processes with a } \\
\text { set correlation structure }\end{array}$ & $+*$ & - & - \\
\hline
\end{tabular}

It should be noted that using any of the presented software solutions requires significant programming skills from the user. Besides, none of them offers the user the possibility to estimate process properties of one's choice, while attempting to implement this manually will take substantially long tome even from an advanced user. An advantage of the "Yuima" package is the option to configure model type and the possibility to simulate several correlated processes simultaneously in case when the process is described by one equation (the example exception is the Heston model).

\section{CONCLUSION}

The literature review offered above demonstrates the successful application of models based on stochastic differential equations to study stochastic processes related to various areas of human activity. Researchers are actively solving a very broad range of practical problems using simulation approach, a flexible method for studying stochastic processes. The current trend in the emerging software products for SDE-based modeling leads to increasing user friendliness, which allows to extend the application of SDE-based models even further, for example into humanities. The analysis of the available software indicated that, currently, the most suitable package for stochastic process simulation is "Yuima", developed in R. However, this software solution still requires its user to possess in-depth knowledge of programming, and does not provide the option to select or configure discretization interval and scheme, 
as well as to estimate the desired properties and characteristics of the process during modeling. Absence of such functionality denies researchers the opportunity to analyze systems quickly, compare existing models, operate and implement various discretization schemes, and, as a consequence, choose the most optimal algorithm for building simulation models.

\section{REFERENCES}

[1] J. Gleick, Chaos: Making a New Science, Viking Books, 1987.

[2] P. Glasserman, Monte Carlo Methods in Financial Engineering, New York: Springer, 2003.

[3] Anne Katrine Duun-Henriksen, M.Sc.,1 Signe Schmidt, M.D.,2 Rikke Meldgaard Røge, M.Sc.,3 Jonas Bech Møller, M.Sc., Ph.D.,3 Kirsten Nørgaard, M.D., D.M.Sc.,2 John Bagterp Jørgensen, M.Sc., Ph.D.,1 and Henrik Madsen, M.Sc., Ph.D.1, "Model Identification Using Stochastic Differential Equation Grey-Box Models in Diabetes," Journal of Diabetes Science and Technology, no. 7(2), pp. 431-440, March 2013.

[4] W.M.H.N. Nilakshi Weerasinghe, Don Kumudu Mallawa Arachchi, "Annual Research Symposium," in Derivation and application of $a$ Stochastic Differential Equation (SDE) Model for cricket, 2013.

[5] Abodolsadeh Neisy and Moslem Peymany, "Financial Modeling by Ordinary and Stochastic Differential Equations," World Applied Sciences Journal, vol. 13, no. 11, pp. 2288-2295, 2011.

[6] S. L. Heston, "A Closed-Form Solution for Options with Stochastic Volatility with Applications to Bond and Currency Options," The Review of Financial Studies, vol. 6, no. 2, 1993.

[7] D. F. Kuznetsov, Stochastic Differential Equations: Theory and Practice of Numerical Solution, Saint-Petersburg: Polytechnical Univ. Press, 2010.

[8] V. Volterra, "Variations and fluctuations of the number of individuals in animal species living together," Animal Ecology, 1931.

[9] S. Kou, Jump-Diffusion Models for Asset Pricing in Financial Engineering, Elsevier, 2008.

[10] F. Rouah, "Euler and Milstein Discretization," [Online]. Available: http://www.frouah.com/pages/finmath.html. [Accessed 2208 2017].

[11] A. Leif, "Efficient Simulation of the Heston Stochastic Volatility Model," Banc of America Securities, December 12,2006.

[12] M. Broadie,Ö. Kaya, "Exact Simulation of Stochastic Volatility and," OPERATIONS RESEARCH, no. 54, pp. 217-231, 2006.

[13] V. Jayanth, Package 'jrvFinance', August 29, 2016.

[14] S. Enrico, "Numerical Methods and Optimization in Finance," 2017, Octomber 19.

[15] S. Iacus, From the sde package to the Yuima Project, Springer, 2008.

[16] "Financial Toolbox," MathWorks, [Online]. Available: https://www.mathworks.com/products/finance.html.

[17] M. J. Aburn, sdeint 0.2.1, 2017.

[18] Hull, John C, Options, Futures and Other Derivatives, 2008. 